\title{
INVARIANT EXTENSION OF QUASICONFORMAL DEFORMATIONS
}

\author{
H. M. REIMANN
}

In a series of papers Ahlfors has studied the differential operator $S$, mapping vectorfields in $\boldsymbol{R}^{\boldsymbol{n}}$ into tensorfields of symmetric tensors with vanishing trace. In particular, he showed that $S$ and its adjoint $S^{*}$ have certain invariance properties with respect to the group of Möbiustransformations. He then proceeded to give an explicit solution for the boundary value problem

$$
\begin{gathered}
\varrho^{-n-2} S^{*} \varrho^{n} S v=0, \quad \varrho(x)=\frac{1}{1-|x|^{2}}, \quad|x|<1, \\
\left.v\right|_{\Sigma}=f, \quad \Sigma=\left\{x \in \boldsymbol{R}^{n}:|x|=1\right\}=\partial B .
\end{gathered}
$$

It should be noted that this is a boundary value problem for vectorfields, involving a hyperbolically invariant second order differential operator. Among other results, he proved that the solutions satisfy the equation

$$
S v(0)_{i j}=\frac{n(n+1)}{2(n-1) \omega_{n}} \int_{\Sigma}\left(f_{i} x_{j}+f_{j} x_{i}\right) d \sigma
$$

provided that $f$ is a tangential vectorfield.

In Chapter 11 of his lecture notes on the geometry and topology of three-manifolds Thurston applied the notion of visual averages to vector- and tensorfields on the sphere $\Sigma \subset \boldsymbol{R}^{\mathbf{3}}$. The visual averages were used for the construction of continuous extensions into $B$ of vectorfields $f$, which were defined on $\Sigma$. The extended vectorfields $v=\operatorname{ext} f$ were shown to satisfy a certain differential equation. Furthermore a formula expressing $S v$ in terms of $\bar{\partial} f$ was derived. It now appears that the two approaches lead to the same result (for dimension $n=3$ ).

The present paper is structured as follows: The first section is introductory. The following two deal with the extension of vectorfields. The reader should have no difficulties in verifying, that the definition for the extension operator given here coincides with the definition given by Thurston.

In the next section, the differential operator $S^{\Sigma}$ on vectorfields on the sphere is introduced and its invariance properties are studied. It is shown that for any tangen- 
tial $C^{1}$-vectorfield $f: \Sigma \rightarrow \boldsymbol{R}^{n}$ the formula

$$
\int_{\Sigma} S^{\Sigma} f_{i j} d \sigma=\left(\frac{n}{2}-\frac{1}{n-1}\right) \int_{\Sigma}\left(x_{i} f_{j}+x_{j} f_{i}\right) d \sigma
$$

holds. The main result can then be expressed by the formula

$$
S v(0)=\frac{n}{n-2} \frac{1}{\omega_{n}} \int_{\Sigma} S^{\Sigma} f d \sigma
$$

which holds for $v=\operatorname{ext} f$. The special case $n=3$ is a different version of Thurston's result. The proof given here should clarify the connection between the two different approaches by Ahlfors and Thurston.

In Sections 7 and 8 the results are applied to the extension problem for quasiconformal mappings. The discussion concentrates on $c$-quasiconformal deformations, i.e. vectorfields $v: \boldsymbol{R}^{n} \rightarrow \boldsymbol{R}^{n}$ satisfying $\|S v\|_{\infty} \leqq c$. These vectorfields have the property that they generate flows of quasiconformal mappings.

In the lowest dimensional case $n=3$, the $K$-quasiconformal mappings $\varphi$ : $\Sigma \rightarrow \Sigma$ can be embedded into a flow of quasiconformal mappings $\varphi_{t}$ generated by a quasiconformal deformation $f$ on $\Sigma$. The extended vectorfield $v=\operatorname{ext} f$ is then a quasiconformal deformation in $B$ and the flow $\psi_{t}$ which it generates gives the desired extension. If $\varphi$ is $K$-quasiconformal then $\psi$ is $K^{3}$-quasiconformal. Still in dimension $n=3$, the same ex ension process can be applied if the quasiconformal mapping $\varphi: \Sigma \rightarrow \Sigma$ is invariant with respect to isomorphic discrete groups $\Gamma$ and $\Lambda$ of Möbiustransformations. It should be noted that the methods of Tukia [6] in connection with the parametrisation of quasiconformal mappings also lead to a proof of this result.

\section{The Möbius group and its Lie-algebra}

The Möbius group $M(n)$ is the group of conformal mappings of the extended euclidean space $\hat{\boldsymbol{R}}^{n}=\boldsymbol{R}^{n} \cup\{\infty\}$. The sutgroup of $M(n)$ which stabilizes the unit ball $B=\left\{x \in \boldsymbol{R}^{n}:|x|<1\right\}$ is isomorphic to $M(n-1)$, it will also be denoted by $M(n-1)$. It is a transformation group of both $B$ and $\Sigma=\partial B$.

The action of the groups $M(n)$ and $M(n-1)$ on the space of $C^{\infty}$-functions $g$ is given by

$$
\tau g(x)=g\left(\tau^{-1} x\right)
$$

and the induced action on the tangentspace can be described by the formula

$$
\tau v(\tau g)=\tau(v g), \quad \tau \in M .
$$

Here, $v$ is a vectorfield on $\hat{\boldsymbol{R}}^{n}, B$ or $\Sigma$ and $v g$ is differentiation of $g$ in direction of the vectorfield $v$. Using euclidean coordinates (these are the only coordinates to be used) and denoting the standard basis in $\boldsymbol{R}^{n}$ by $\left\{e_{1}, \ldots, e_{n}\right\}$, the action can be described by 
the formulas

$$
\begin{gathered}
v(x)=\sum_{i=1}^{n} v_{i}(x) e_{i}, \quad x \in \boldsymbol{R}^{n}, \\
\tau v(x)=T\left(\tau^{-1} x\right) v\left(\tau^{-1} x\right), \quad \tau \in M(n),
\end{gathered}
$$

where $T=\left(t_{i j}\right), t_{i j}(x)=\left(\partial t_{i} / \partial x_{j}\right)(x)$, is the Jacobian matrix of the conformal mapping $\tau=\left(\tau_{1}, \ldots, \tau_{n}\right)$. Since we are mainly interested in the action of $M(n-1)$ on $B$ and on $\Sigma$, we can refrain from introducing a coordinate system in a neighbourhood of the point $\infty$.

An element in the Lie algebra of the Möbius group is a vectorfield generating a 1-parameter subgroup of Möbiustransformations. The Lie algebra $m(n-1)$ of the Möbius group $M(n-1)$ acting on $B$ or on $\Sigma$ has a basis given by the vectorfields

$$
\begin{gathered}
\ell^{k}(x)=\frac{1+|x|^{2}}{2} e_{k}-x_{k} \sum_{j=1}^{n} x_{j} e_{j}, \quad k=1, \ldots, n, \\
\ell^{i j}(x)=x_{i} e_{j}-x_{j} e_{i}, \quad 1 \leqq i<j \leqq n .
\end{gathered}
$$

The Lie bracket (Poisson bracket) is given by

$$
\begin{gathered}
{[v, w] g=v(w g)-w(v g), \quad v, w \in m(n-1),} \\
{[v, w]_{j}(x)=\sum_{i=1}^{n}\left(v_{i} \frac{\partial w_{j}}{\partial x_{i}}-w_{i} \frac{\partial v_{j}}{\partial x_{i}}\right) .}
\end{gathered}
$$

In particular

$$
\left[\ell^{k}, \ell^{n}\right]=\ell^{k n}, \quad k<n
$$

The group $M(n-1)$ acts on its Lie algebra

$$
v \rightarrow \tau v, \quad \tau \in M(n-1), \quad v \in m(n-1) .
$$

This action is usually referred to as the adjoint representation.

If $\tau_{s}$ is the 1-parameter group generated by the vectorfield $\ell^{n}$, then it follows from the above commutator relation (by exponentiation) that

$$
\begin{aligned}
& \tau_{s} \ell^{k}=C h s \ell^{k}-S h s \ell^{k n}, \quad k<n, \\
& \tau_{s} \ell^{n}=\ell^{n} .
\end{aligned}
$$

\section{Extension of vectorfields}

For a continuous vectorfield $f$ on $\Sigma$ which is tangent to the sphere

$$
(x, f)(x)=\sum_{j=1}^{n} x_{j} f_{j}(x)=0, \quad|x|=1,
$$

the extension $v=\operatorname{ext} f$ is defined by

$$
\begin{gathered}
\tau(\operatorname{ext} f)(0)=\frac{n}{n-1} \frac{1}{\omega_{n}} \int_{\Sigma} \tau f(x) d \sigma(x), \quad \tau \in M(n-1), \\
\omega_{n}=\int_{\Sigma} d \sigma \quad\left(\Sigma \subset \boldsymbol{R}^{n}\right) .
\end{gathered}
$$


First it is to be noted, that for $\tau$ in the stabilizer of the origin, i.e. for $\tau$ a rotation, the formula does not lead to a contradiction. In fact, setting $v=\operatorname{ext} f, \tau x=K x$ with $K$ an orthogonal matrix, it follows that

$$
\begin{gathered}
\tau v(0)=K v(0)=c_{n} K \int_{\Sigma} f(x) d \sigma(x) \\
=c_{n} \int_{\Sigma} \tau f(\tau x) d \sigma(x)=c_{n} \int_{\Sigma} \tau f(y) d \sigma(y) .
\end{gathered}
$$

Therefore, if $\tau \in M(n-1)$ is a Möbiustransformation mapping $y$ onto 0 , then the vectorfield $v=\operatorname{ext} f$ at $y$ is unambiguously determined by

$$
T(y) v(y)=\tau v(0) .
$$

Furthermore it follows, that for any $\varrho \in M(n-1)$

$$
\varrho(\operatorname{ext} f)=\operatorname{ext}(\varrho f) \text {. }
$$

The extension operator can be represented in the form of a Poisson-type integraloperator. In the remainder of this section, the corresponding kernel is calculated explicitly.

Since the transformations $\tau \in M(n-1)$ are conformal, the scalar product

$$
\frac{1}{\omega_{n}} \int_{\Sigma}(g(x), f(x)) d \sigma(x)
$$

of two tangential vectorfields on the sphere satisfies

$$
\begin{gathered}
\frac{1}{\omega_{n}} \int_{\Sigma}(\tau g(x), \tau f(x)) d \sigma(x)=\frac{1}{\omega_{n}} \int_{\Sigma}\left(\operatorname{det} T\left(\tau^{-1} x\right)\right)^{2 / n}\left(g\left(\tau^{-1} x\right), f\left(\tau^{-1} x\right)\right) d \sigma(x) \\
\left.=\frac{1}{\omega_{n}} \int_{\Sigma}(\operatorname{det} T(y))\right)^{(n+1) / n}(g(y), f(y)) d \sigma(y) .
\end{gathered}
$$

Conformality is expressed by the fact that $T(y)$ is a multiple of an orthogonal matrix. The transformation $\tau$ is considered as a transformation of $\hat{\boldsymbol{R}}^{n}$. The expression $(\operatorname{det} T(y))^{(n-1) / n}$ is the Poisson kernel for the hyperbolically invariant Laplace operator. If $\tau \in M(n-1)$ satisfies $\tau(z)=0$ then

$$
\operatorname{det} T(y)=\left(\frac{1-|z|^{2}}{|y-z|^{2}}\right)^{n} \quad y \in \Sigma .
$$

Observing that for tangential vectorfields $f=\left(f_{1}, \ldots, f_{n}\right)$

$$
\int_{\Sigma} f_{k} d \sigma(x)=\int_{\Sigma}\left(\ell^{k}, f\right)(x) d \sigma(x)
$$

one is led to the formulas $\left(c_{n}=\frac{n}{(n-1) \omega_{n}}\right)$

$$
\begin{gathered}
T(z) v(z)=\tau v(0)=c_{n} \sum_{k=1}^{n} \ell^{k}(0) \int_{\Sigma}\left(\ell^{k}, \tau f\right)(x) d \sigma(x), \\
v(z)=c_{n} \sum_{k=1}^{n}\left(\tau^{-1} \ell^{k}\right)(z) \int_{\Sigma}\left(\tau^{-1} \ell^{k}, f\right)(y)\left(\frac{1-|z|^{2}}{|y-z|^{2}}\right)^{n+1} d \sigma(y) .
\end{gathered}
$$


If $\tau_{s}$ is the 1-parameter group generated by the Lie algebra vectorfield $\ell^{n}$, then

$$
\tau_{s}(0)=\frac{S h s}{1+C h s} e_{n} .
$$

In order to calculate $v$ at the point

$$
z=r e_{n}=\frac{S h s}{1+C h s} e_{n}
$$

it is sufficient to know the action of $\left(\tau_{s}\right)^{-1}=\tau_{-s}$ on the Lie algebra vectorfields $\ell^{k}$ :

$$
\begin{gathered}
\tau_{-s} \ell^{k}(x)=C h s \ell^{k}(x)+\operatorname{Shs} \ell^{k n}(x), \quad k<n, \\
\tau_{-s} \ell^{n}(x)=\ell^{n}(x) .
\end{gathered}
$$

A short calculation gives

$$
\tau_{-s} \ell^{k}(z)=\frac{1-r^{2}}{2} e_{k}, \quad k=1, \ldots, n,
$$

and for $y \in \Sigma$

$$
\begin{gathered}
\left(\tau_{-s} \ell^{k}, f\right)=\operatorname{Chs} f_{k}+\operatorname{Sh} s\left(y_{k} f_{n}-y_{n} f_{k}\right) \\
=\frac{1+r^{2}}{1-r^{2}} f_{k}+\frac{2}{1-r^{2}}\left(y_{k}(z, f)-f_{k}(z, y)\right), \quad k<n, \\
\left(\tau_{-s} \ell^{n}, f\right)=f_{n},
\end{gathered}
$$

where we have used that $C h s=\left(1+r^{2}\right) /\left(1-r^{2}\right), S h s=2 r /\left(1-r^{2}\right)$. If $c$ is a unit vector orthogonal to $z$, then

$$
(c, v(z))=c_{n} \int_{\Sigma}\left\{(c, f) \frac{1+r^{2}}{2}+(c, y)(z, f)-(c, f)(z, y)\right\}\left(\frac{1-r^{2}}{|z-y|^{2}}\right)^{n+1} d \sigma(y)
$$

whereas for $c=e_{n}$

$$
\left(e_{n}, v(z)\right)=c_{n} \frac{1-r^{2}}{2 r} \int_{\Sigma}(z, f)\left(\frac{1-r^{2}}{|z-y|^{2}}\right)^{n+1} d \sigma(y) .
$$

It is now easy to verify that these two formulas can be expressed in matrix form by Ahlfors' formula ([1], p. 34):

with

$$
v(z)=\frac{n}{2(n-1)} \frac{1}{\omega_{n}} \int_{\Sigma}|z-y|^{2}(I-2 Q(z-y)) f(y)\left(\frac{1-|z|^{2}}{|z-y|^{2}}\right)^{n+1} d \sigma(y)
$$

In fact, if $(c, z)=0$ then

$$
Q(x)_{i j}=\frac{x_{i} x_{j}}{|x|^{2}}
$$

$$
(c, v(z))=\frac{c_{n}}{2} \int_{\Sigma}\left\{(c, f)|z-y|^{2}+2(z, f)(c, y)\right\}\left(\frac{1-|z|^{2}}{|y-z|^{2}}\right)^{n+1} d \sigma(y)
$$

and

$$
\left(e_{n}, v(z)\right)=\frac{c_{n}}{2 r} \int_{\Sigma}\left\{(z, f)|z-y|^{2}-2(z, z-y)(z, f)\right\}\left(\frac{1-|z|^{2}}{|y-z|^{2}}\right)^{n+1} d \sigma(y) .
$$




\section{Properties of the extension}

Assume that $f: \Sigma \rightarrow \boldsymbol{R}^{n}$ is a continuous vectorfield on the sphere $\Sigma \subset \boldsymbol{R}^{n}$, which is tangent

$$
(x, f(x))=0, \quad|x|=1 .
$$

The extension $v=\operatorname{ext} f$ of $f$ is defined by

$$
\tau v(0)=\frac{n}{n-1} \frac{1}{\omega_{n}} \int_{\Sigma} \tau f(x) d \sigma(x), \quad \tau \in M(n-1),
$$

or equivalently by Ahlfors' formula

$$
v(z)=\frac{n}{2(n-1)} \frac{1}{\omega_{n}} \int_{\Sigma}(I-2 Q(z-y)) f(y) \frac{\left(1-|z|^{2}\right)^{n+1}}{|y-z|^{2 n}} d \sigma(y) .
$$

Proposition 1. The extension $v=\operatorname{ext} f$ is a continuous extension of $f$ :

$$
\lim _{z \rightarrow x} v(z)=f(x), \quad x \in \Sigma .
$$

This is a result of Alhfors ([1], Theorem 1). For the dimension $n=3$ a direct proof is given by Thurston ([5], Proposition 11.1.1).

The differential operator $S$ mapping vectorfields into fields of symmetric tensors with vanishing trace is defined by

$$
(S v)_{i j}=\frac{1}{2}\left(\frac{\partial v_{i}}{\partial x_{j}}+\frac{\partial v_{j}}{\partial x_{i}}\right)-\frac{\delta_{i j}}{n} \sum_{k=1}^{n} \frac{\partial v_{k}}{\partial x_{k}} .
$$

The adjoint operator $S^{*}$ is then given by

$$
\left(S^{*} \varphi\right)_{i}=\sum_{j=1}^{n} \frac{\partial \varphi_{i j}}{\partial x_{j}} .
$$

Proposition 2 (Ahlfors [1], Theorem 1). The extension $v=\operatorname{ext} f$ is the unique solution with boundary values $f$ of the invariant differential equation

$$
\varrho^{-n-2} S^{*} \varrho^{n} S v=0, \quad \varrho(x)=\frac{1}{1-|x|^{2}} .
$$

Corollary. If $f$ is the restriction of the Lie algebra vectorfield $\ell \in m(n-1)$ to the sphere, then $\ell=\operatorname{ext} f$.

It suffices to observe that $S \ell=0$.

Proposition 3.

$$
S v(0)_{i j}=\frac{n(n+1)}{2(n-1)} \frac{1}{\omega_{n}} \int_{\Sigma}\left(x_{i} f_{j}+x_{j} f_{i}\right) d \sigma(x) .
$$

This is a special case of Theorem 3 in Ahlfors [1]. It can be proved directly from the definition of $v=\operatorname{ext} f$. 


\section{The definition for $S^{\Sigma}$}

First order differential operators on the unit sphere $\Sigma \subset \boldsymbol{R}^{n}$ are given by tangential vectorfields. Let us in particular consider the vectorfields $\ell^{k}$ from the Lie algebra of $M(n-1)$

$$
\ell^{k}(x)=\sum_{j=1}^{n}\left(\delta_{j k}-x_{j} x_{k}\right) e_{j}, \quad|x|=1 .
$$

Definition. The differential operator $S^{\Sigma}$ mapping tangential vectorfields $f$ on $\Sigma$ into functions $S^{\Sigma} f$ with values in the space of symmetric matrices of vanishing trace is defined by

$$
S^{\Sigma} f(x)_{i j}=\frac{1}{2}\left(\ell^{i} f, \ell^{j}\right)+\frac{1}{2}\left(\ell^{j} f, \ell^{i}\right)-\frac{\left(\ell^{i}, \ell^{j}\right)}{n-1} \sum_{k=1}^{n}\left(\ell^{k} f, \ell^{k}\right) .
$$

The expression $\ell^{i} f$ is the derivative of the vectorfield $f$ in direction of $\ell^{i}$ (not the Lie bracket):

$$
\ell^{i} f(x)_{j}=\sum_{k=1}^{n} \frac{\partial f_{j}}{\partial x_{k}}\left(\delta_{j k}-x_{j} x_{k}\right) .
$$

Let us note the following equations for the scalar products:

$$
\begin{gathered}
\left(\ell^{i}, \ell^{j}\right)=\delta_{i j}-x_{i} x_{j}, \\
\sum_{k=1}^{n}\left(\ell^{k}, \ell^{k}\right)=\sum_{k=1}^{n}\left(1-x_{k}^{2}\right)=n-1 .
\end{gathered}
$$

At $x=-e_{n}$ the vectorfields $\ell^{i}$ are given by

$$
\begin{gathered}
\ell^{i}\left(-e_{n}\right)=e_{i} \quad i=1, \ldots, n-1 \\
\ell^{n}\left(-e_{n}\right)=0 .
\end{gathered}
$$

At this point the operator $S^{\Sigma}$ therefore takes the form

$$
S^{\Sigma} F\left(-e_{n}\right)_{i j}=\left\{\begin{array}{l}
\frac{1}{2}\left(\frac{\partial f_{i}}{\partial x_{j}}+\frac{\partial f_{j}}{\partial x_{i}}\right)-\frac{\delta_{i j}}{n} \sum_{k=1}^{n-1} \frac{\partial f_{k}}{\partial x_{j}}, \quad i \neq n, j \neq n, \\
0 \text { if } i=n \text { or if } j=n .
\end{array}\right.
$$

Theorem 1. The operator $S^{\Sigma}$ has the following invariance property with respect to Möbiustransformations $\tau \in M(n-1)$ :

$$
S^{\Sigma} \tau f(x)=T\left(\tau^{-1} x\right) S^{\Sigma} f\left(\tau^{-1}\right) T^{-1}\left(\tau^{-1} x\right)
$$

for any tangential $C^{1}$-vectorfield $f$ on $\Sigma$.

Let us first prove the invariance under rotations $\tau x=T x$, where $T=\left(t_{i j}\right)$ is an orthogonal matrix. A straightforward calculation shows that

$$
\left(\ell^{i} \tau f, \ell^{k}\right)(x)=\sum_{j, m} t_{i m}\left(\ell^{m} f, \ell^{j}\right)\left(\tau^{-1} x\right) t_{k j} .
$$

In particular this gives

$$
\sum_{k=1}^{n}\left(\ell^{k} \tau f, \ell^{k}\right)(x)=\sum_{k=1}^{n}\left(\ell^{k} f, \ell^{k}\right)\left(\tau^{-1} x\right) .
$$


Furthemore, since $\left(\ell^{i}, \ell^{j}\right)=\delta_{i j}-x_{i} x_{j}$ one has

$$
\left(\ell^{i}, \ell^{k}\right)(x)=\sum_{j, m} t_{i n i}\left(\ell^{m}, \ell^{j}\right) t_{k j} .
$$

Those equations clearly imply invariance under rotations.

Next let us introduce stereographic projection as the restriction to $\Sigma$ of the Möbiustransformation $\pi \in M(n)$ given by

$$
\begin{gathered}
(\pi x)_{i}=\frac{2 x_{i}}{|x|^{2}-2 x_{n}+1}, \quad i=1, \ldots, n-1, \\
(n x)_{n}=\frac{1-|x|^{2}}{|x|^{2}-2 x_{n}+1}, \quad i=n .
\end{gathered}
$$

This transformation is often referred to as the Cayley transformation. It induces a bijection between tangential vectorfields on the punctured sphere and tangential vectorfields on $\boldsymbol{R}^{n-1}=\partial \boldsymbol{R}_{+}^{n}$. If $P(x)=\left(p_{i j}(x)\right)$ is the Jacobian matrix of $\pi$, then a tangential vectorfield $f=\left(f_{1}, \ldots, f_{n}\right)$ on $\Sigma$ corresponds to the vectorfield $\tilde{f}=$ $\left(\tilde{f}_{1}, \ldots, \tilde{f}_{n-1}, 0\right)=\pi f$ given by

$$
\tilde{f}(x)=P\left(\pi^{-1} x\right) f\left(\pi^{-1} x\right) .
$$

A short consideration shows that the partial derivatives at $-e_{n}$ and 0 respectively satisfy

Furthermore

$$
\frac{\partial f_{i}}{\partial x_{j}}\left(-e_{n}\right)=\frac{\partial \tilde{f}_{i}}{\partial x_{j}}(0), \quad i, j=1, \ldots, n-1
$$

$$
P\left(-e_{n}\right)=\frac{1}{2} I \text {. }
$$

Consider the $S$-operator on $\boldsymbol{R}^{n-1}$ and set for any $C^{1}$-vectorfield $\tilde{f}=$ $\left(f_{1}, \ldots, f_{n-1}, 0\right)$

$$
\tilde{S} \tilde{f}_{i j}=\left\{\begin{array}{l}
\frac{1}{2}\left(\frac{\partial f_{i}}{\partial x_{i}}+\frac{\partial f_{j}}{\partial x_{i}}\right)-\frac{\delta_{i j}}{n-1} \sum_{k=1}^{n} \frac{\partial f_{k}}{\partial x_{k}}, \quad i \neq n, \quad j \neq n, \\
0 \text { if } i=n \text { or if } j=n .
\end{array}\right.
$$

If $\alpha \in M(n)$ is a conformal mapping, which maps the upper halfspace $\boldsymbol{R}_{+}^{n}$ onto itself, then its Jacobian matrix $A=\left(a_{i j}\right)$ at $x \in \boldsymbol{R}^{n-1}=\partial \boldsymbol{R}_{+}^{n}$ satisfies

$$
\begin{gathered}
a_{i n}=a_{n i}=0, \quad i=1, \ldots, n-1, \\
a_{n n}=(\operatorname{det} A)^{1 / n},
\end{gathered}
$$

since $A$ is a multiple of an orthogonal matrix leaving invariant the hyperplane $\left(x, e_{n}\right)$ $=0$. The known invariance properties of the $S$-operator (see Ahlfors [1], equation 1.7) admit the conclusion that

$$
\tilde{S} \alpha \tilde{f}(x)=A\left(\alpha^{-1} x\right) \tilde{S} \tilde{f}\left(\alpha^{-1} x\right) A^{-1}\left(\alpha^{-1} x\right), \quad x \in \boldsymbol{R}^{n-1} .
$$

The proof of the theorem can now be completed as follows: Because $S^{\Sigma}$ is invariant under rotations it suffices to prove the theorem under the special assump- 
tion that $x=\tau x=-e_{n}$. From the above equations it then follows that

$$
\tilde{S} \tilde{f}(0)=P\left(-e_{n}\right) S^{\Sigma} f\left(-e_{n}\right) P^{-1}\left(-e_{n}\right) .
$$

If $\tau$ is conjugate to the transformation $\alpha$,

then

$$
\tau=\pi^{-1} \circ \alpha \circ \pi,
$$

$$
\begin{gathered}
S^{\Sigma} \tau f\left(-e_{n}\right)=P^{-1}\left(-e_{n}\right) \tilde{S} \pi^{-1} \alpha \tilde{f}(0) P\left(-e_{n}\right) \\
=P^{-1}\left(-e_{n}\right) A(0) P\left(-e_{n}\right) S^{\Sigma} f\left(-e_{n}\right) P^{-1}\left(-e_{n}\right) A(0)^{-1} P\left(-e_{n}\right) \\
=T\left(-e_{n}\right) S^{\Sigma} f\left(-e_{n}\right) T^{-1}\left(-e_{n}\right) .
\end{gathered}
$$

Corollary. Under stereographic projection $\pi$ the operator $S^{\Sigma}$ corresponds to the operator $\tilde{S}$

$$
\tilde{S} \tilde{f}(x)=P\left(\pi^{-1} x\right) S^{\Sigma} f\left(\pi^{-1} x\right) P^{-1}\left(\pi^{-1} x\right) .
$$

\section{Partial integration on $\Sigma$}

Lemma 1.

$$
\int_{\Sigma} g(x) x_{i} d \sigma(x)=\frac{1}{n-1} \int_{\Sigma} \ell^{i} g(x) d \sigma(x)
$$

for any $C^{\mathbf{1}}$-function $g$ on $\Sigma$.

The proof is based on explicit calculations using polar coordinates

$$
\begin{gathered}
x_{1}=\sin \theta_{1} \ldots \sin \theta_{n-1}, \\
\ldots \\
x_{n-1}=\sin \theta_{1} \cos \theta_{2} \\
x_{n}=\cos \theta_{1}, \\
\int_{\Sigma} g x_{n} d \sigma_{n}=\int_{\Sigma^{n-1}} d \sigma_{n-1} \int_{0}^{\pi} g \cos \theta_{1}\left(\sin \theta_{1}\right)^{n-2} d \theta_{1} \\
=-\int_{\Sigma^{n-1}} d \sigma_{n-1} \int_{0}^{\pi} \frac{\partial g}{\partial \theta_{1}} \frac{1}{n-1}\left(\sin \theta_{1}\right)^{n-1} d \theta_{1}=-\frac{1}{n-1} \int_{\Sigma} \frac{\partial g}{\partial \theta_{1}} \sin \theta_{1} d \sigma_{n}, \\
\frac{\partial g}{\partial \theta_{1}}=\frac{\partial g}{\partial x_{1}} \cos \theta_{1} \sin \theta_{2} \ldots \sin \theta_{n-1}+\ldots+\frac{\partial g}{\partial x_{n-1}} \cos \theta_{1} \cos \theta_{2}-\frac{\partial g}{\partial x_{n}} \sin \theta_{1},
\end{gathered}
$$

$\sin \theta_{1} \frac{\partial g}{\partial \theta_{1}}=\frac{\partial g}{\partial x_{1}} x_{1} x_{n}+\ldots+\frac{\partial g}{\partial x_{n-1}} x_{n-1} x_{n}-\frac{\partial g}{\partial x_{n}}\left(1-x_{n}^{2}\right)=-\sum_{k=1}^{n}\left(\delta_{k n}-x_{k} x_{n}\right) \frac{\partial g}{\partial x_{k}}$.

Lemma 2.

$$
\int_{\Sigma}\left(\ell^{i} f, \ell^{j}\right) d \sigma=\int_{\Sigma}\left((n-1) f_{j} x_{i}+x_{j} f_{i}\right) d \sigma
$$


for any tangential $C^{1}$-vectorfield on $\Sigma$.

Proof. We have

$$
\begin{gathered}
\ell^{i}\left(x_{j} x_{k}\right)=x_{k}\left(\delta_{i j}-x_{i} x_{j}\right)+x_{j}\left(\delta_{i k}-x_{i} x_{k}\right), \\
0=\ell^{i}\left(\sum_{k=1}^{n} x_{j} x_{k} f_{k}\right)=\sum_{k=1}^{n} x_{j} x_{k} \ell^{i} f_{k}+\sum_{k=1}^{n} f_{k} \ell^{i}\left(x_{j} x_{k}\right)=\sum_{k=1}^{n} x_{j} x_{k} \ell^{i} f_{k}+x_{j} f_{i}, \\
\left(\ell^{i} f, \ell^{j}\right)=\ell^{i} f_{j}-\sum_{k=1}^{n} x_{j} x_{k} \ell^{i} f_{k}=\ell^{i} f_{j}+x_{j} f_{i} .
\end{gathered}
$$

The proof of Lemma 2 is hereby reduced to the statement of Lemma 1.

Lemma 3.

$$
\int_{\Sigma} S^{\Sigma} f_{i j} d \sigma=\frac{(n-2)(n+1)}{2(n-1)} \int_{\Sigma}\left(x_{i} f_{j}+x_{j} f_{i}\right) d \sigma
$$

for any tangential $C^{1}$-vectorfield $f$ on $\Sigma$.

There remains the calculation of the integrals over the expressions

$$
\left(\ell^{i}, \ell^{j}\right) \sum_{k=1}^{n}\left(\ell^{k} f, \ell^{k}\right)=\left(\delta_{i j}-x_{i} x_{j}\right) \sum_{k=1}^{n}\left(\ell^{k} f_{k}+x_{k} f_{k}\right) .
$$

First note that

$$
\int_{\Sigma} \sum_{k=1}^{n}\left(\ell^{k} f, \ell^{k}\right) d \sigma=\int_{\Sigma} \sum_{k=1}^{n} \ell^{k} f_{k} d \sigma=(n-1) \int_{\Sigma} \sum_{k=1}^{n} x_{k} f_{k} d \sigma=0 .
$$

Furthermore

$$
\begin{gathered}
\int_{\Sigma} x_{i} x_{j} \sum_{k=1}^{n} \ell^{k} f_{k} d \sigma=\int_{\Sigma} \sum_{k=1}^{n}\left[\ell^{k}\left(x_{i} x_{j} f_{k}\right)-f_{k} x_{i}\left(\delta_{k j}-x_{k} x_{j}\right)-f_{k} x_{j}\left(\delta_{i k}-x_{i} x_{k}\right)\right] d \sigma \\
=\int_{\Sigma}\left(-f_{j} x_{i}-f_{i} x_{j}\right) d \sigma
\end{gathered}
$$

which shows that

$$
\int_{\Sigma}\left(\ell^{i}, \ell^{j}\right) \sum_{k=1}^{n}\left(\ell^{k} f, \ell^{k}\right) d \sigma=\int_{\Sigma}\left(x_{i} f_{j}+x_{j} f_{i}\right) d \sigma .
$$

From Lemma 2 it then follows that

$$
\int_{\Sigma} S^{\Sigma} f_{i j} d \sigma=\left(\frac{n}{2}-\frac{1}{n-1}\right) \int_{\Sigma}\left(x_{i} f_{j}+x_{j} f_{i}\right) d \sigma
$$

\section{The main result}

In the previous section it was shown that for tangential $C^{\mathbf{1}}$-vectorfields on $\Sigma$ the formula

$$
\int_{\Sigma} S^{\Sigma} f_{i j} d \sigma=\frac{(n-2)(n+1)}{2(n-1)} \int_{\Sigma}\left(x_{i} f_{j}+x_{j} f_{i}\right) d \sigma
$$

holds. Since this formula is based on partial integration, it still holds if the tangential vectorfield $f$ only has distributional derivatives (in the direction of the vectorfields 
$\left.\ell^{i}, i=1, \ldots, n\right)$ which are integrable. In connection with Ahlfors' formula

$$
S v(0)_{i j}=\frac{n(n+1)}{2(n-1)} \frac{1}{\omega_{n}} \int_{\Sigma}\left(x_{i} f_{j}+x_{j} f_{i}\right) d \sigma
$$

this result means that we have proved the following theorem

Theorem 2. Assume that the continuous vectorfield $f: \Sigma \rightarrow \boldsymbol{R}^{n}, n \geqq 3$, is tangent to $\Sigma$ and has integrable distributional derivatives. Then the extension $v=\operatorname{ext} f$ satisfies

$$
S v(0)=\frac{n}{n-2} \frac{1}{\omega_{n}} \int_{\Sigma} S^{\Sigma} f(x) d \sigma .
$$

For the case $n=3$ this theorem (in a different form) is due to Thurston ([5], Proposition 11.1.5).

Corollary 1. If $\tau \in M(n-1)$ is a Möbiustransformation, mapping the unit ball onto itself and the point $z$ into the origin, then

$$
S v(z)=T(z)^{-1} \frac{n}{n-2} \frac{1}{\omega_{n}} \int_{\Sigma} T(y) S^{\Sigma} f(y) T^{-1}(y)\left(\frac{1-|z|^{2}}{|z-y|^{2}}\right)^{n-1} d \sigma(y) T(z)
$$

$(T(x)$ is the Jacobian matrix of $\tau$ at $x)$.

If the theorem is applied to $\tau f$, the corollary follows from the invariance relations for $S$ and $S^{\Sigma}$.

\section{Quasiconformal deformations}

A continuous vectorfield $v: \boldsymbol{R}^{n} \rightarrow \boldsymbol{R}^{n}$ is a $c$-quasiconformal deformation, if it has locally integrable distributional derivatives and if

$$
\|S v\|_{\infty}=\sup _{x \in R^{n}}|S v(x)| \leqq c<\infty .
$$

In the present context, the norm of an $n \times n$ matrix $A$ is defined by

$$
|A|=\sup _{|y|=1}|A y| \text {. }
$$

Observe that if $T$ is a multiple of an orthogonal matrix, then

$$
\left|T A T^{-1}\right|=|A| \text {. }
$$

For dimensions $n \geqq 3$ the only conformal deformations (i.e. the 0-quasiconformal deformations) are the vectorfields in the Lie algebra of $M(n)$. Furthermore any quasiconformal deformation in $\boldsymbol{R}^{n}$ decomposes as the sum of a conformal deformation and a quasiconformal deformation satisfying

$$
v(x)=O(|x| \log |x|), \quad|x| \rightarrow \infty
$$


(Sarvas [4]). This last statement is also true for $n=2$. In this case the conformal deformations are given by the analytic functions. The condition

$$
\|S f\|_{\infty} \leqq c
$$

can be written in complex notation $f=f_{1}+i f_{2}$ as

$$
\left\|f_{\bar{z}}\right\|_{\infty}=\left\|\left(\begin{array}{cc}
\operatorname{Re} f_{\bar{z}} & \operatorname{Im} f_{\bar{z}} \\
\operatorname{Im} f_{\bar{z}} & -\operatorname{Re} f_{\bar{z}}
\end{array}\right)\right\|_{\infty} \leqq c,
$$

and the equation $f_{\bar{z}}=g$ with $\|g\|_{\infty} \leqq c$ admits a continuous solution $f$ with $f(z)=$ $O(|z| \log |z|)$.

In view of the corollary to Theorem 1 , the $c$-quasiconformal deformations on the sphere $\Sigma \subset \boldsymbol{R}^{n}, n \geqq 3$, can now be defined as the continuous tangential vectorfields $f$ with integrable distributional derivatives satisfying

$$
\left\|S^{\Sigma} f\right\|_{\infty}=\sup _{x \in \Sigma} \operatorname{ess}\left|S^{\Sigma} f(x)\right| \leqq c<\infty .
$$

If $f$ is such a vectorfield, then under stereographic projection it is mapped onto a vectorfield

satisfying

$$
\tilde{f}=\left(\tilde{f_{1}}, \ldots, \tilde{f}_{n-1}\right)
$$

$$
\|S \tilde{f}\|_{\infty}=\left\|S^{\Sigma} f\right\|_{\infty} .
$$

Conversely, if $\tilde{f}$ is a $c$-quasiconformal deformation on $\boldsymbol{R}^{n-1}$ which satisfies $\tilde{f}(x)=O(|x| \log |x|)$, then it is the image under stereographic projection of a quasiconformal deformation $f$ on $\Sigma$. The only difficulty arising is the singularity at $e_{n}$. However, by the results of Sarvas [4] this is a removable singularity.

Needless to say, the Lie algebra vectorfields are mapped isomorphically under stereographic projection.

Corollary 2. If $f: \Sigma \rightarrow \boldsymbol{R}^{n}$ is a c-quasiconformal deformation, then $v=\operatorname{ext} f$ : $B \rightarrow \boldsymbol{R}^{n}$ is a $(n c /(n-2))$-quasiconformal deformation $(n \geqq 3)$.

Let $\tau \in M(n-1)$ be a Möbiustransformation such that $\tau z=0$. Then

$$
\begin{aligned}
& |S v(z)|=\left|T(z) S v(z) T(z)^{-1}\right|=|S \tau v(0)|=\frac{n}{n-2} \frac{1}{\omega_{n}}\left|\int_{\Sigma} S^{\Sigma} \tau f(x) d \sigma(x)\right| \\
& \leqq \frac{n}{n-2} \frac{1}{\omega_{n}} \int_{\Sigma}\left|T\left(\tau^{-1} x\right) S^{\Sigma} f\left(\tau^{-1} x\right) T\left(\tau^{-1} x\right)\right| d \sigma(x) \leqq \frac{n}{n-2} \frac{1}{\omega_{n}} \int_{\Sigma} c d \sigma .
\end{aligned}
$$

We will need the following theorem [3]:

Suppose that $v(x, t)$ is a c-quasiconformal deformation in the sense that

a) $v(x, t)$ is continuous on $\boldsymbol{R}^{n} \times[0,1]$ and has locally integrable distributional derivatives,

b)

c)

$$
\begin{gathered}
v(x, t)=O(|x| \log |x|), \quad|x| \rightarrow \infty, \\
\|S v(\cdot, t)\|_{\infty} \leqq c .
\end{gathered}
$$


Denote the solutions of the differential equation

$$
\frac{d}{d t} x=v(x, t)
$$

with initial condition $x(0)=z$ by $\varphi(z, t)$. Then for fixed $t, \varphi(z, t)$ is a $e^{2 c t}$-quasiconformal mapping.

For the special case $n=2$ this theorem has a converse, known as the parametric representation of quasiconformal mappings (see e.g. [2]):

If $\varphi: C \rightarrow C$ is a $K$-quasiconformal mapping, then there exists a $((\log K) / 2)$ quasiconformal deformation $v(x, t)$ such that the flow $\varphi(z, t)$ as defined above satisfies $\varphi(z, 1)=\varphi(z)$.

Corollary 3. A K-quasiconformal mapping of $\Sigma \subset \boldsymbol{R}^{\mathbf{3}}$ onto itself extends to a $K^{3}$-quasiconformal mapping of the unit ball $B \subset \boldsymbol{R}^{3}$.

If $\varphi: \Sigma \rightarrow \Sigma$ is a $K$-quasiconformal mapping, which we assume to be normalized by $\varphi\left(e_{n}\right)=e_{n}$, then there exists a $((\log K) / 2)$-quasiconformal deformation $\tilde{f}(x, t)$ : $\boldsymbol{R}^{2} \times[0,1] \rightarrow \boldsymbol{R}^{2}$ whose flow $\tilde{\varphi}(x, t)$ satisfies

$$
\tilde{\varphi}(x, 1)=\pi \circ \varphi \circ \pi^{-1}(x) .
$$

The transformed vectorfield $\pi^{-1} \tilde{f}=f$ is then a $((\log K) / 2)$-quasiconformal deformation on $\Sigma$ (with a parameter $t$ ) and can therefore be extended to a $((3 \log K) / 2)$ quasiconformal deformation $v=\operatorname{ext} f$ on $B$. If as above $\psi(z, t)$ is the solution of the differential equation $(d / d t) x=v(x, t)$ with initial condition $\psi(z, 0)=z \in B$, then

$$
\psi(z)= \begin{cases}\psi(z, 1), & z \in B, \\ \varphi(z), & z \in \Sigma,\end{cases}
$$

is a $K^{3}$-quasiconformal extension of $\varphi$.

\section{Group invariant extensions of quasiconformal mappings}

Theorem 3. Assume that $\theta: \Gamma \rightarrow \Lambda$ is an isomorphism between groups of $M \ddot{o}-$ biustransformations $\Gamma, \Lambda \subset M(2)$, acting on the unit ball $\bar{B}=B \cup \Sigma$ in $\boldsymbol{R}^{3}$. Furthermore, assume that $\varphi: \Sigma \rightarrow \Sigma$ is a K-quasiconformal mapping satisfying

$$
\varphi \circ \gamma=\theta(\gamma) \circ \varphi \text { for all } \gamma \in \Gamma \text {. }
$$

Then $\varphi$ extends to a $K^{3}$-quasiconformal mapping $\psi: \bar{B} \rightarrow \bar{B}$ such that

$$
\psi \circ \gamma=\theta(\gamma) \circ \psi \text { for all } \gamma \in \Gamma \text {. }
$$

Before giving a proof of this theorem let us first recall some well known facts about quasiconformal mappings $\varphi: \hat{\boldsymbol{C}} \rightarrow \hat{\boldsymbol{C}}$ and their parametric representations. 
Assume that $\varphi$ is normalized by $\varphi(0)=0, \varphi(1)=1, \varphi(\infty)=\infty$ and denote its dilatation by $\mu$. If $\lambda \circ \varphi=\varphi \circ \gamma$ for some Möbiustransformations $\lambda, \gamma$, then

$$
\mu(y)=\frac{\gamma^{\prime}}{\bar{\gamma}^{\prime}}\left(\gamma^{-1} y\right) \mu\left(\gamma^{-1} y\right)
$$

as can be seen immediately by differentiating the conjugation relation.

Conversely, if the complex dilatation $\mu$ of the normalized quasiconformal mapping $\varphi$ satisfies

$$
\mu(y)=\frac{\gamma^{\prime}}{\bar{\gamma}^{\prime}}\left(\gamma^{-1} y\right) \mu\left(\gamma^{-1} y\right)
$$

where $\gamma$ is a Möbiustransformation, then the mapping $\lambda$ defined by

is a Möbiustransformation.

$$
\lambda \circ \varphi=\varphi \circ \gamma
$$

The parametric representation $\varphi(z, t)$ for the normalized quasiconformal mapping $\varphi$ is constructed as follows: Define $\mu(z, t)$ by the equations

$$
\begin{gathered}
\frac{1+|\mu(z, t)|}{1-|\mu(z, t)|}=\left(\frac{1+|\mu(z)|}{1-|\mu(z)|}\right)^{t}, \quad 0 \leqq t \leqq 1, \\
\arg \mu(z, t)=\arg \mu(z) .
\end{gathered}
$$

Then $\varphi(z, t)$ is the unique normalized solution of the Beltrami equation

$$
\varphi_{\bar{z}}=\mu(z, t) \varphi_{z} \cdot
$$

If now $\lambda \circ \varphi=\varphi \circ \gamma$, then not only $\mu(z)$ but also $\mu(z, t)$ will satisfy the equation

$$
\mu(y, t)=\frac{\gamma^{\prime}}{\bar{\gamma}^{\prime}}\left(\gamma^{-1} y\right) \mu\left(\gamma^{-1} y, t\right) .
$$

Hence there exist Möbiustransformations $\lambda_{t}$ such that $\varphi_{t}(z)=\varphi(z, t)$ satisfies

$$
\lambda_{t} \circ \varphi_{t}=\varphi_{t} \circ \gamma .
$$

The quasiconformal deformation associated to the parametric representation is the vectorfield defined by

$$
f(\varphi(z, t), t)=\frac{d}{d t} \varphi(z, t)
$$

If $\lambda_{t} \circ \varphi_{t}=\varphi_{t} \circ \gamma$, then it satisfies

$$
\begin{gathered}
f\left(\varphi_{t} \circ \gamma\right)=\frac{d}{d t} \varphi_{t} \circ \gamma=\frac{d}{d t} \lambda_{t} \circ \varphi_{t}=\frac{d \lambda_{t}}{d t} \circ \varphi_{t}+\lambda_{t}^{\prime} \circ \varphi_{t} \frac{d}{d t} \varphi_{t}, \\
f\left(\lambda_{t}(x), t\right)=\frac{d \lambda_{t}}{d t}(x)+\lambda_{t}^{\prime}(x) f(x, t) .
\end{gathered}
$$

Observe now that the mappings $\lambda_{t}$ are Möbiustransformations. Hence the vector- 
field $g$ defined by

$$
g\left(\lambda_{t}(x), t\right)=\frac{d \lambda_{t}}{d t}(x)
$$

is in the Lie algebra of the Möbiusgroup (acting on $\hat{\boldsymbol{C}}$ ). Using the notation $\lambda_{t} f$ for the vectorfield $f$ transformed by the Möbiustransformation $\lambda_{t}$, we find that

$$
\begin{gathered}
\lambda_{t} f(w, t)=\lambda_{t}^{\prime}(x) f(x, t) \quad\left(w=\lambda_{t}(x)\right), \\
f(w, t)=g(w, t)+\lambda_{t} f(w, t) .
\end{gathered}
$$

For the proof of Theorem 3 it can be assumed that the $K$-quasiconformal mapping $\varphi: \Sigma \rightarrow \Sigma$ is normalized by the requirement that $e_{1}, e_{3}$ and $-e_{3}$ are fixed points. Consider then the conjugate $K$-quasiconformal mapping $\tilde{\varphi}=\pi \circ \varphi \circ \pi^{-1}: \hat{\boldsymbol{C}} \rightarrow \hat{\boldsymbol{C}}$ and the conjugate groups $\tilde{\Gamma}=\pi \circ \Gamma \circ \pi^{-1}, \tilde{\Lambda}=\pi \circ \Lambda \circ \pi^{-1}$ and construct the associated parametric representation and vectorfields $\tilde{f}(x, t), \tilde{g}(x, t)$. These vectorfields can be mapped onto tangential vectorfields on the sphere such that one arrives at the following situation: There exists a $((\log K) / 2)$-quasiconformal deformation $f_{t}(x)=f(x, t)$ and a 1-parameter family of quasiconformal mappings $\varphi_{t}(z)=\varphi(z, t)$ of the sphere such that

$$
\begin{gathered}
\frac{d}{d t} \varphi(z, t)=f(\varphi(z, t), t), \\
\varphi(z, 1)=\varphi(z) .
\end{gathered}
$$

Furthermore there exist groups $\Gamma_{t}$ and Lie algebra vectorfields $g_{t}(x)=g(x, t)$ such that

$$
f_{t}=g_{t}+\lambda_{t} f_{t}, \quad t \in[0,1]
$$

$\left(\Delta_{0}=\Gamma, \Lambda_{1}=\Lambda\right.$ and $g_{t}$ depends on $\left.\gamma\right)$.

The extended vectorfields

then satisfy

$$
\begin{aligned}
& v_{t}=\operatorname{ext} f_{t}, \\
& u_{t}=\operatorname{ext} g_{t}
\end{aligned}
$$

$$
v_{t}=u_{t}+\lambda_{t} v_{t}
$$

where $\lambda_{t} v_{t}$ is the vectorfield $v_{t}$ transformed by the Möbiustransformation $\lambda_{t}$ acting on $B$. Furthermore, $u_{t}$ is a Lie algebra vectorfield (conformal deformation) and one must have

$$
\frac{d \lambda_{t}}{d t}(z)=u_{t}\left(\lambda_{t}(z), t\right), \quad z \in B
$$

since this equation holds on $\Sigma$.

By Corollary $2, v$ is a $((3 \log K) / 2)$-quasiconformal deformation. The $K^{3 t}$ quasiconformal mappings $\psi_{t}: B \rightarrow B$, which are solutions to the equation

$$
\frac{d}{d t} \psi_{t}(z)=v\left(\psi_{t}(z), t\right)
$$


with initial condition $\psi_{0}(z)=z$ are extensions of $\varphi_{t}$. It must be shown that

$$
\lambda_{t} \circ \psi_{t}=\psi_{t} \circ \gamma \text {. }
$$

The mappings $\chi_{t}=\lambda_{t} \circ \psi_{t}$ satisfy the differential equation

$$
\frac{d}{d t} \chi_{t}(z)=\frac{d \lambda_{t}}{d t}(x)+\lambda_{t} v\left(\lambda_{t}(x), t\right), \quad x=\psi_{t}(z),
$$

as can be seen by differentiating with respect to $t$ and applying the chain rule. Since

and

$$
\frac{d \lambda_{t}}{d t}(x)=u\left(\lambda_{t}(x), t\right)
$$

$$
v(w, t)=u(w, t)+\lambda_{t} v(w, t)
$$

it follows that $\chi_{t}$ satisfies the differential equation

and the initial condition

$$
\frac{d}{d t} \chi_{t}(z)=v\left(\chi_{t}(z), t\right)
$$

$$
\chi_{0}(z)=\lambda_{0} \circ \psi_{0}(z)=\gamma(z) .
$$

However, the function $\psi_{t} \circ \gamma(z)$ satisfies the same differential equation with the same initial condition. Since the solutions are unique, it follows that

$$
\psi_{t} \circ \gamma=\lambda_{t} \circ \psi_{t} .
$$

\section{References}

[1] AHLFORs, L. V.: Invariant operators and integral representations in hyperbolic space. - Math. Scand. 36, 1975, 27-43.

[2] Gehring, F. W., and E. Reich: Area distortion under quasiconformal mappings. - Ann. Acad. Sci. Fenn. Ser. A I Math. 388, 1966, 1-15.

[3] Reimann, H. M.: Ordinary differential equations and quasiconformal mappings. - Invent. Math. 33, 1976, 247-270.

[4] SARVAS, J.: Singularities of quasiconformal deformations in $\boldsymbol{R}^{n}$. - Indiana Univ. Math. J. 31, $1982,121-134$.

[5] Thurston, W. P.: The geometry and topology of three-manifolds. - Lecture notes, Princeton University, 1978-79.

[6] TukiA, P.: Quasiconformal extension of quasisymmetric mappings compatible with a Möbius group. - Acta Math. (to appear).

University of Bern

Department of Mathematics

Sidlerstr. 5

$\mathrm{CH}-3012$ Bern

Switzerland

Received 2 March 1984 